\title{
Perspectivas futuras de la TC cardíaca: ¿Herramienta todo en uno?
}

\author{
Future perspectives of cardiac CT: One-stop shop tool?
}

\author{
Gastón A. Rodríguez Granillo
}

\section{RESUMEN}

La angiografía coronaria por tomografía computada multidetector (ACTCM) ha emergido como una alternativa a la cinecoronariografía en pacientes con probabilidad intermedia de enfermedad coronaria. Sin embargo, existe una limitada correlación entre enfermedad obstructiva detectada por ACTCM y la presencia de isquemia. Por lo tanto, en los últimos años se ha hecho énfasis en la búsqueda de una herramienta no invasiva "todo en uno" que proporcione información integral tanto anatómica como funcional/fisiológica. Para dicho propósito, la ACTCM ofrece dos estrategias: la perfusión miocárdica por TC bajo estrés farmacológico y la evaluación funcional a partir de datos anatómicos (reserva fraccional del flujo por TC y el cálculo del gradiente de atenuación transluminal).

Palabras clave: tomografía multislice, imágenes cardiovasculares, enfermedad coronaria.

\begin{abstract}
Computed tomography coronary angiography (CTCA) has emerged as a surrogate of invasive angiography in patients with moderate probability of obstructive coronary artery disease. However, there is a limited correlation between the extent of coronary obstruction detected by CTCA and the presence of ischemia. This has lead to a increased interest in the search of a one-stop shop non-invasive diagnostic tool that would be able to provide during a single session a comprehensive analysis regarding both anatomical and functional information. For this purpose, CTCA offers two strategies: stress myocardial CT perfusion; and functional evaluation obtained from anatomical data (fractional flow reserve by $\mathrm{CT}$ and calculation of the transluminal attenuation gradient)
\end{abstract}

Keywords: multislice tomography, cardiovascular imaging, coronary disease.

Revista Argentina de Cardioangiología Intervencionista 2014;5(2):122-124

Entre los años 2000 y 2010, el número de muertes por enfermedad cardiovascular (ECV) se redujo un $31 \%$. A pesar de estos avances y de la intensificación de estrategias de prevención y tratamiento, en el año 2010, dentro de los Estados Unidos, un tercio de las muertes en adultos fueron causadas por ECV; una persona muere por ECV cada 40 segundos, y por un evento coronario cada 83 segundos, demandando un constante perfeccionamiento de las herramientas de detección precoz, sin soslayar el costo-beneficio. ${ }^{1}$ Los métodos de diagnóstico por imagen cardíaca no invasivos pueden ser divididos en anatómicos (angiografía coronaria por tomografía computada multidetector, ACTCM; angiorresonancia magnética coronaria) y funcionales (ecocardiografía con estrés, medicina nuclear, resonancia magnética con estrés farmacológico). A su vez, po-

1. Sanatorio Otamendi. CABA, Argentina. Consejo de Investigaciones Científcas y Técnicas (CONICET).

$\triangle$ Correspondencia: grodriguezgranillo@gmail.com

Conflictos de intereses: no existen.

Enviado: 24-1-2014 | Aceptado: 15-2-2014 dría hacerse una disquisición entre métodos que evalúan la enfermedad coronaria (el compromiso luminal) y aquellos que evalúan la enfermedad aterosclerótica coronaria (el compromiso parietal + luminal).

Dentro de este contexto, y apoyada en evidencia robusta acerca de su valor diagnóstico y pronóstico, la ACTCM ha emergido como una alternativa a la cinecoronariografía en pacientes con probabilidad intermedia de enfermedad coronaria, particularmente en presencia de pruebas funcionales no concluyentes. ${ }^{2}$

Sin embargo, numerosos estudios han documentado la limitada correlación entre enfermedad obstructiva detectada por ACTCM y la presencia de alteración significativa de la perfusión miocárdica, ya sea detectada por medicina nuclear, por reserva fraccional de flujo (RFF) o por otros métodos. Del mismo modo, puede observarse isquemia en ausencia de lesiones mayores del $50 \% .^{3}$

Por lo tanto, en los últimos años se ha hecho énfasis en la búsqueda de una herramienta no invasiva "todo en uno" que proporcione información integral tanto anatómica como funcional/fisiológica. ${ }^{4,5}$

Para dicho propósito, la ACTCM ofrece dos estrategias: 1) perfusión miocárdica por TC bajo estrés far- 
macológico, y 2) evaluación funcional a partir de datos anatómicos $\left(\mathrm{RFF}_{\mathrm{TC}}\right.$, y el cálculo del gradiente de atenuación transluminal), que se discutirán brevemente a continuación.

\section{PERFUSIÓN MIOCÁRDICA PORTC}

La enorme evolución de los equipos disponibles, incluyendo tomógrafos de doble fuente (TDF), de doble energía (TDE), y equipos volumétricos de gran cobertura, hacen posible la valoración de la perfusión miocárdica durante el mismo estudio de ACTCM. Existen distintas técnicas (helicoidal vs. dinámica), protocolos de adquisición (estrés-reposo $v s$. reposo-estrés), y fármacos utilizados (dipiridamol, adenosina, regadenoson), que escapan al objetivo de este breve artículo.

Existen en la literatura alrededor de 20 ensayos clínicos que evaluaron la perfusión miocárdica por TC, incluyendo diferentes poblaciones y protocolos de adquisición.5 En términos generales, se ha demostrado que la combinación de perfusión miocárdica por TC y la ACTCM permite mejorar significativamente el rendimiento diagnóstico del método, presentando la perfusión en sí misma valores de sensibilidad y especificidad cercanos al $90 \%$ y resultados similares al SPECT y a la RM con estrés. ${ }^{5}$

La principal limitación que presenta la perfusión miocárdica por TC es la elevada dosis de radiación, particularmente en adquisiciones dinámicas mediante movimiento alternante de la mesa (con TDF). Sin embargo, deben destacarse los enormes avances que se han logrado en la implementación de técnicas de reducción de dosis, y en este punto se hace referencia también a la ACTCM. En este respecto, a las conocidas adquisiciones mediante gatillado prospectivo, modulación de la corriente del tubo y reducción del voltaje, se han agregado nuevas y efectivas técnicas como la adquisición con pitch alto, y la reconstrucción iterativa. Estas medidas han logrado reducir la dosis de radiación de la ACTCM reposo-esfuerzo a niveles similares al SPECT reposo-esfuerzo; y la dosis de ACTCM en reposo a niveles muy bajos $(1 \mathrm{a} 4 \mathrm{mSv})$. $^{5}$

Si bien cada institución suele adoptar un protocolo para sistematizar los estudios, los mismos deberían ajustarse a la probabilidad pretest de cada paciente. En pacientes con pretest elevado sería razonable priorizar la perfusión, realizando entonces el estrés en primera instancia; mientras que en pacientes con probabilidad pretest baja a intermedia debería realizarse el reposo en primera instancia y prescindir entonces de la adquisición con estrés en caso de ausencia de enfermedad.

En cuanto a perspectivas futuras, es factible que en los próximos años mejoras en la resolución temporal permitan la valoración anatómica y funcional con adquisiciones únicamente en estrés en pacientes con pretest elevado, prescindiendo de la adquisición en reposo y expandiendo las indicaciones a este grupo de pacientes.
Debe mencionarse que actualmente la implementación de protocolos de inyección de contraste bi- o trifásicos y particularmente la utilización de TDE permiten reducir significativamente el volumen de contraste. Asimismo, los TDE parecen ofrecer una solución a los artefactos de endurecimiento del haz, que suelen generar hipodensidades miocárdicas falsamente sugestivas de defectos de perfusión. ${ }^{6}$

\section{EVALUACIÓN FUNCIONAL A PARTIR DE DATOS ANATÓMICOS: RESERVA FRACCIO- NAL DE FLUJO (RFF) Y GRADIENTE DE ATE- NUACIÓN TRANSLUMINAL}

Como se mencionó con anterioridad, la correlación entre el grado de obstrucción luminal de las lesiones y su impacto funcional (hemodinámico) es relativamente pobre. Asimismo, la perfusión miocárdica por TC requiere generalmente dos adquisiciones (estrés y reposo), y por lo tanto una dosis de radiación mayor. En consecuencia, existe gran interés en la obtención de información hemodinámica directamente a partir de datos anatómicos fruto una única exploración.

La TC ofrece actualmente para este propósito dos alternativas: la reserva fraccional de flujo coronario (RFF) y gradiente de atenuación transluminal. El escenario ideal para este tipo de exploraciones sería utilizando equipos de gran cobertura (320 filas) que permitan obtener la totalidad del volumen cardíaco en un único latido.

La serie de estudios DEFER y FAME demostró el beneficio de la angioplastia guiada por RFF $v$ s. la guiada por la anatomía, conduciendo a la evaluación invasiva de la RFF durante la cinecoronariografía a convertirse en el estándar de referencia para la valoración de la significancia hemodinámica de las estenosis coronarias. ${ }^{7,8}$ $\mathrm{Al}$ determinar las características del flujo proximal y distal a las lesiones en condiciones de hiperemia inducida por fármacos, la RFF brinda importante información fisiológica y, utilizada de forma apropiada, reduce el número de intervenciones innecesarias.

El avance en el estudio de técnicas de dinámica de fluidos computacional utilizado con frecuencia en el desarrollo de aviones y automóviles tiene un gran potencial, y estudios recientes han demostrado resultados prometedores en cuanto a la evaluación no invasiva de la RFF por TC $\left(\mathrm{RFF}_{\mathrm{TC}}\right)$. El primer estudio multicéntrico prospectivo al respecto (DISCOVER-FLOW) arrojó resultados intrigantes, mejorando significativamente el área bajo la curva para la detección de isquemia vs. la exploración anatómica aislada. ${ }^{9}$ No obstante, en otro estudio multicéntrico de mayor tamaño (DeFACTO), la RFF ${ }_{\mathrm{TC}}$ no incrementó la especificidad del la información anatómica. ${ }^{10}$ Un estudio muy reciente reportó una interesante potencial aplicación clínica de la $\mathrm{RFF}_{\mathrm{TC}}$, como herramienta no invasiva que permita predecir el resultado funcional del tratamiento percutáneo mediante el stenting virtual. ${ }^{11}$ Se necesitan más 
estudios para llegar a una conclusión acerca de esta novedosa tecnología. Deben destacarse sin embargo algunas limitaciones de la $\mathrm{RFF}_{\mathrm{TC}}$, particularmente un posprocesamiento muy complejo y el hecho de que para su cálculo se asumen demasiadas premisas no necesariamente ciertas; entre otras, que tanto el miocardio como la función microvascular se encuentran indemnes.
Finalmente, el gradiente de atenuación transluminal se origina en el concepto de que el tiempo de tránsito del contraste a través de una estenosis debe ser distinto del tiempo de tránsito a través de un vaso sin estenosis. Sin embargo, los resultados obtenidos hasta la actualidad han sido relativamente pobres, con escaso valor incremental sobre los datos anatómicos aislados. ${ }^{12}$

\section{BIBLIOGRAFÍA}

1. Go AS, Mozaffarian D, Roger VL, et al. Heart disease and stroke statistics-2014 update: a report from the American Heart Association. Circulation 2013 Jan 1;127(1):143-52.

2. Taylor AJ, Cerqueira M, Hodgson JM, Mark D, Min J, O'Gara P, et al. ACCFI SCCT/ACR/AHA/ASE/ASNC/NASCI/SCAI/SCMR 2010 appropriate use criteria for cardiac computed tomography. A report of the American College of Cardiology Foundation Appropriate Use Criteria Task Force, the Society of Cardiovascular Computed Tomography, the American College of Radiology, the American Heart Association, the American Society of Echocardiography, the American Society of Nuclear Cardiology, the North American Society for Cardiovascular Imaging, the Society for Cardiovascular Angiography and Interventions, and the Society for Cardiovascular Magnetic Resonance. J Am Coll Cardiol 2010 Nov 23:56(22):1864-1894.

3. Rossi A, Papadopoulou SL, Pugliese F, Russo B, Dharampal AS, Dedic A, Kitslaar PH, Broersen A, Meijboom WB, van Geuns RJ, Wragg A, Ligthart J, Schultz C, Petersen SE, Nieman K, Krestin GP, de Feyter PJ. Quantitative CT Coronary Angiography: Does It Predict Functionally Significant Coronary Stenoses?. Circ Cardiovasc Imaging 2013 Nov 26 [Epub ahead of print].

4. Rodriguez-Granillo GA, Ingino C, Lylyk P. Myocardial perfusion imaging and infarct characterization using multidetector cardiac computed tomography. World J Cardiol 2010 July 26; 2(7):198-204.

5. Bastarrika G, De Cecco CN, Jiménez-Juan L, Zavodni A, Schoepf UJ. Reserva de flujo coronario y perfusión miocárdica por TC. En: Rodríguez Granillo GA, Gómez E, Bastarrika G, Cademartiri F (editores). TC y RM Cardiovascular:Fundamentos Clínicos. Buenos Aires: Ediciones Journal;2014.p. 157-168.

6. Rodríguez-Granillo GA, Rosales MA, Degrossi E, Rodriguez AE. Signal density of left ventricular myocardial segments and impact of beam hardening artifact: implications for myocardial perfusion assessment by multidetector CT coronary angiography. Int J Cardiovasc Imaging 2010 Mar;26(3):345-354.

7. Pijls NH, van Schaardenburgh P, Manoharan G, Boersma E, Bech JW, van't Veer M, Bar F, Hoorntje J, Koolen J, Wijns W, de Bruyne B. Percutaneous coronary intervention of functionally nonsignificant stenosis: 5-year foIlow-up of the DEFER Study. J Am Coll Cardiol 2007;49:2105-2111.

8. Tonino PA, De BB, Pijls NH, Siebert U, Ikeno F, van't Veer M, Klauss V, Manoharan G, Engstrom T, Oldroyd KG, Ver Lee PN, MacCarthy PA, Fearon WE. FAME Study Investigators: Fractional flow reserve versus angiography for guiding percutaneous coronary intervention. NEngl J Med 2009;360:213224.

9. Koo BK, Erglis A, Doh JH, Daniels DV, Jegere S, Kim HS, et al. Diagnosis of ischemia-causing coronary stenoses by noninvasive fractional flow reserve computed from coronary computed tomographic angiograms. Results from the prospective multicenter DISCOVER-FLOW (Diagnosis of Ischemia-Causing Stenoses Obtained Via Noninvasive Fractional Flow Reserve) study. J Am Coll Cardiol 2011 Nov 1;58(19):1989-1997.

10. Min JK, Leipsic J, Pencina MJ, Berman DS, Koo BK, van Mieghem C, et al. Diagnostic accuracy of fractional flow reserve from anatomic CT angiography. JAMA 2012 Sep 26;308(12):1237-1245.

11. Kim KH, Doh JH, Koo BK, Min JK, Erglis A, Yang HM, Park KW, Lee HY, Kang HJ, Kim YJ, Lee SY, Kim HS. A novel noninvasive technology for treatment planning using virtual coronary stenting and computed tomography-derived computed fractional flow reserve. JACC Cardiovasc Interv. 2013 Dec 5. pii: S1936-8798(13)01540-9. doi: 10.1016/j.jcin.2013.05.024. [Epub ahead of print].

12. Yoon YE, Choi JH, Kim JH, Park KW, Doh JH, Kim YJ, et al. Noninvasive diagnosis of ischemia-causing coronary stenosis using CT angiography: diagnostic value of transluminal attenuation gradient and fractional flow reserve computed from coronary CT angiography compared to invasively measured fractional flow reserve. JACC Cardiovasc Imaging 2012 Nov:5(11):1088-1096. 